\title{
The global dynamics for an age-structured SLIS model with the delay
}

\author{
Hongwu Tan ${ }^{1}$, Xin Yang ${ }^{1}$ and Hui Cao ${ }^{1 *}$ (C)
}

\section{"Correspondence:}

caohui@sust.edu.cn

'Department of Mathematics, Shaanxi University of Science and

Technology, Xi'an, P.R. China

\begin{abstract}
This paper deals with the global dynamics for a SLIS epidemic model with infection age. In our model, we also consider the time delay in the progression from the latent individuals to becoming infectious individuals. We verify the well posedness of the model by changing it into an abstract nondensely defined Cauchy problem and find conditions for the existence of disease free equilibrium and endemic equilibrium. The theoretic analysis shows that the disease-free equilibrium is globally asymptotically stable as the basic reproduction number $R_{0}$ is less than unity and that the endemic equilibrium is locally asymptotically stable and the system is uniformly persistent as $R_{0}$ is greater than unity. The numerical simulations illustrate that the endemic equilibrium may be asymptotically stable as $R_{0}>1$.
\end{abstract}

Keywords: Age-structured model; The delay; $C_{0}$-semigroup; Stability; Uniform persistence

\section{Introduction}

Iin 1927, Kermack and McKendrick [1] proposed the SIR infectious disease model framework in which the total population was divided into three classes referred to as susceptible, infective, and recovered, and assumed that the recovered individuals have the permanent immunity. However, SIR infectious disease model framework cannot describe epidemics with latency, such as HIV/AIDS, tuberculosis (TB), hepatitis B, malaria, and so on. It leads to the appearance of the SLIR models discussing the spread of the epidemic diseases with latency, where "L" represents the latent individuals [2-8].

In fact, many sexually transmitted diseases such as gonorrhea and chlamydial infections are known to result in a little or no acquired immunity after recovery [9]. For the diseases that cause a very brief immune for the recovered individuals, the immune period may be ignored, and the model under study may be a suitable approximation [10]. That is, compared with SLIR and SLIRS epidemic models, the SLIS models are more suitable to describe the infectious diseases with latency and short immune period.

For the SLIS epidemic models, the outflow of the latent individuals is often described by an ordinary differential equation

$$
\frac{d L}{d t}=-(d+\alpha) L
$$

(c) The Author(s) 2020. This article is licensed under a Creative Commons Attribution 4.0 International License, which permits use, sharing, adaptation, distribution and reproduction in any medium or format, as long as you give appropriate credit to the original author(s) and the source, provide a link to the Creative Commons licence, and indicate if changes were made. The images or other third party material in this article are included in the article's Creative Commons licence, unless indicated otherwise in a credit line to the material. If material is not included in the article's Creative Commons licence and your intended use is not permitted by statutory regulation or exceeds the permitted use, you will need to obtain permission directly from the copyright holder. To view a copy of this licence, visit http://creativecommons.org/licenses/by/4.0/. 
where $L=L(t)$ denotes the number of the latent individuals at time $t, d$ is the natural death rate, and $\alpha$ is the progression rate from latent individuals to infectious individuals. The assumption in model (1.1) implies that the progression rate $\alpha$ is subject to the exponential distribution. However, for some diseases (such as TB, HIV/AIDS, etc.), the realistic situation is that the latent individuals must stay in the latent class for some time before becoming the infectious class, and after that time, the progression rate from latent individuals to infectious individuals depends on the infection age of the latent individuals. Namely, the progression rate $\alpha$ in (1.1) should be replaced with the following piecewise function:

$$
\alpha(a)= \begin{cases}\alpha_{*}(a), & a>\tau, \\ 0, & a \leq \tau,\end{cases}
$$

where $\alpha(a) \in L_{+}^{\infty}((0,+\infty), \mathbb{R}), \tau \geq 0$ represents the shortest time required from initial infection to becoming infectious class (i.e. the shortest latency period), and $a$ is the period of time that the latent individuals stay in the latent class since the initial infection, which is referred to be the infection age. Thus, by incorporating the infection age and applying (1.2), (1.1) becomes

$$
\frac{\partial L(t, a)}{\partial t}+\frac{\partial L(t, a)}{\partial a}=-(d+\alpha(a)) L(t, a), \quad t \geq 0, a \geq 0,
$$

where $L(t, a)$ is the density of latent individuals with respect to infection age $a$ at time $t$.

Let $S=S(t)$ denote the number of the susceptible individuals at time $t, I=I(t)$ denote the number of the infectious individuals at time $t, \Lambda>0$ be the constant recruitment rate of susceptible individuals, $\beta>0$ be the rate at which susceptible individuals become infected by an infectious individual, and $\gamma>0$ be the recovery rates of infectious individuals. Based on the progression rate $\alpha(a)$ and equation (1.3), for the latent individuals with infection age, we build an age-structured SLIS epidemic model with the delay progression rate as follows:

$$
\left\{\begin{array}{l}
\frac{d S(t)}{d t}=\Lambda-d S(t)-\beta S(t) I(t)+\gamma I(t), \\
\frac{\partial L(t, a)}{\partial t}+\frac{\partial L(t, a)}{\partial a}=-d L(t, a)-\alpha(a) L(t, a), \quad t \geq 0, a \geq 0 \\
\frac{d I(t)}{d t}=\int_{0}^{+\infty} \alpha(a) L(t, a) d a-d I(t)-\mu I(t)-\gamma I(t), \quad t \geq 0 \\
L(t, 0)=\beta S(t) I(t), \quad t \geq 0
\end{array}\right.
$$

with the initial conditions

$$
S(0)=S_{0} \geq 0, \quad L(0, a)=L_{0}(a) \in L_{+}^{1}(0,+\infty), \quad I(0)=I_{0} \geq 0 .
$$

Our interest in this paper is investigating the effects of time delay and age structure on the dynamics of SLIS epidemic model. This paper is organized as follows. In Sect. 2, we present some preliminary results and the well-posedness of system (1.4). In Sect. 3, we prove the existence of equilibria, especially the existence and uniqueness of endemic equilibrium for the linearized system of (1.4) around the equilibria. In Sect. 4, we prove the global stability of disease free equilibrium $E_{0}$ and the local stability of the endemic equilibrium $E_{*}$. In Sect. 5, we discuss the uniform persistence of the infection. These methods and 
technique used in these sections are similar to those in [11]. Finally, we give a discussion and conclusions in Sect. 6.

\section{Preliminaries and well-posedness}

In this part, we first give some preliminaries on linear operators and $C_{0}$-semigroup theory and some notations. And then, we rewrite system (1.4) as an abstract equation on a suitable Banach lattice. Finally, we establish the well-posedness result for system (1.4) and prove the nonnegativity and boundedness of solutions.

Let $P: D(P) \subset X \rightarrow X$ be a linear operator on a Banach space $X$. Denote by $\rho(P)$ the resolvent set of $P$. The spectrum of $P$ is defined as $\sigma(P)=\mathbb{C} \backslash \rho(P)$. The point spectrum of $P$ is the set

$$
\sigma_{p}(P):=\{\lambda \in \mathbb{C}: N(\lambda I-P) \neq\{0\}\}
$$

Definition 2.1 Let $P: D(P) \subseteq X \rightarrow X$ be a linear operator. If there exist real constants $M \geq 1$ and $\omega \in \mathbb{R}$ such that $(\omega,+\infty) \subseteq \rho(P)$ and

$$
\left\|(\lambda-P)^{-n}\right\| \leq \frac{M}{(\lambda-\omega)^{n}} \quad \text { for all } n \in \mathbb{N}_{+} \text {and } \lambda>\omega,
$$

then the linear operator $(P, D(P))$ is called a Hille-Yosida operator.

For Hille-Yosida operators, we have the following perturbation results.

Lemma 2.1 (see $[12,13])$ Let $(A, D(A))$ be a Hille-Yosida operator on a Banach space $X$, and let $B \in \mathscr{L}(X)$, where $\mathscr{L}(X)$ denotes the set of all bounded linear operators on $X$. Then the sum $C=A+B$ is a Hille-Yosida operator as well.

For a Hille-Yosida operator $(P, D(P))$ on a Banach space $X$, we set

$$
\begin{aligned}
& X_{0}:=(\overline{D(P)},\|\cdot\|), \\
& D\left(P_{0}\right):=\left\{x \in D(P): P x \in X_{0}\right\}, \\
& P_{0} x:=P x \quad \text { for } x \in D\left(P_{0}\right) .
\end{aligned}
$$

The operator $\left(P_{0}, D\left(P_{0}\right)\right)$ is called the part of $P$ in $X_{0}$, and the following result holds.

Lemma 2.2 (see $[12,13])$ If $(P, D(P))$ is a Hille-Yosida operator, then its part $\left(P_{0}, D\left(P_{0}\right)\right)$ generates a $C_{0}$-semigroup $\left(T_{0}(t)\right)_{t \geq 0}$ on $X_{0}$.

Now we set about to rewrite system (1.4) into an abstract evolution equation. Let

$$
X=\mathbb{R} \times L^{1}((0,+\infty), \mathbb{R}) \times \mathbb{R} \times \mathbb{R}
$$

and define the linear operator $\mathcal{A}: D(\mathcal{A}) \subseteq X \longrightarrow X$ by

$$
\mathcal{A}\left(\begin{array}{l}
x \\
y \\
z \\
0
\end{array}\right)=\left(\begin{array}{c}
-d x \\
-\frac{d y}{d a}-(d+\alpha(a)) y \\
-(d+\mu+\gamma) z \\
-y(0)
\end{array}\right)
$$


with $D(\mathcal{A})=\mathbb{R} \times W^{1,1}((0,+\infty), \mathbb{R}) \times \mathbb{R} \times\{0\}$. It is clear that $\overline{D(\mathcal{A})}=\mathbb{R} \times L^{1}((0,+\infty), \mathbb{R}) \times$ $\mathbb{R} \times\{0\}$, and thus $D(\mathcal{A})$ is not dense in $X$. Define the nonlinear map $\mathcal{F}: \overline{D(\mathcal{A})} \rightarrow X$ by

$$
\mathcal{F}\left(\begin{array}{l}
x \\
y \\
z \\
0
\end{array}\right)=\left(\begin{array}{c}
\Lambda-\beta x z+\gamma z \\
0 \\
\int_{0}^{+\infty} \alpha(a) y(a) d a \\
\beta x z
\end{array}\right)
$$

It is clear that $\mathcal{F}$ is Lipschitz continuous. Let $u(t)=(S(t), L(t, \cdot), I(t), 0)^{T}$. We can rewrite system (1.4) as the following abstract Cauchy problem:

$$
\left\{\begin{array}{l}
\frac{\mathrm{d}}{\mathrm{d} t}(u(t))=\mathcal{A} u(t)+\mathcal{F}(u(t)), \quad t \geq 0, \\
u(0)=u_{0}
\end{array}\right.
$$

with $u_{0}=\left(S_{0}, L_{0}(a), I_{0}, 0\right)^{T}$. Further, we have

$$
u(t)=u_{0}+\mathcal{A} \int_{0}^{t} u(s) d s+\int_{0}^{t} \mathcal{F}(u(s)) d s .
$$

Let

$$
\begin{aligned}
& X_{0}=\overline{D(\mathcal{A})}=\mathbb{R} \times L^{1}((0,+\infty), \mathbb{R}) \times \mathbb{R} \times\{0\}, \\
& X_{0+}=\mathbb{R}_{+} \times L_{+}^{1}((0,+\infty), \mathbb{R}) \times \mathbb{R}_{+} \times\{0\}, \\
& \Omega:=\{\lambda \in \mathbb{C}: \operatorname{Re}(\lambda)>-d\} .
\end{aligned}
$$

We have the following result.

Theorem 2.1 The operator $(\mathcal{A}, D(\mathcal{A}))$ is a Hille-Yosida operator.

Proof For $(\phi, \varphi, \omega, \psi) \in X,(\tilde{\phi}, \tilde{\varphi}, \tilde{\omega}, 0) \in D(\mathcal{A})$, and $\lambda \in \Omega$, we have

$$
(\lambda-\mathcal{A})^{-1}\left(\begin{array}{c}
\phi \\
\varphi \\
\omega \\
\psi
\end{array}\right)=\left(\begin{array}{c}
\tilde{\phi} \\
\tilde{\varphi} \\
\tilde{\omega} \\
0
\end{array}\right) \Leftrightarrow\left\{\begin{array}{l}
(\lambda+d) \tilde{\phi}=\phi \\
\tilde{\varphi}^{\prime}=-(\lambda+d+\alpha(a)) \tilde{\varphi}+\varphi \\
(\lambda+d+\mu+\gamma) \tilde{\omega}=\omega \\
\tilde{\varphi}(0)=\psi
\end{array}\right.
$$

It means that

$$
\left\{\begin{array}{l}
\tilde{\phi}=\frac{\phi}{\lambda+d}, \\
\tilde{\varphi}=e^{-\int_{0}^{a}(\lambda+d+\alpha(\theta)) d \theta} \psi+\int_{0}^{a} e^{\int_{a}^{s}(\lambda+d+\alpha(\theta)) d \theta} \varphi(s) d s, \\
\tilde{\omega}=\frac{\omega}{\lambda+d+\mu+\gamma} .
\end{array}\right.
$$

Integrating the second equation of (2.3) with respect to the age variable $a$, and adding all the equations, we obtain that

$$
|\tilde{\phi}|+\|\tilde{\varphi}\|_{L^{1}}+|\tilde{\omega}| \leq \frac{1}{\lambda+d}\left(|\phi|+\|\varphi\|_{L^{1}}+|\omega|+|\psi|\right) .
$$


That is,

$$
\left\|(\lambda-\mathcal{A})^{-1}\right\| \leq \frac{1}{\lambda+d} \quad \text { for all } \lambda \in \Omega
$$

which implies that $(\mathcal{A}, D(\mathcal{A}))$ is a Hille-Yosida operator.

By using of Lemma 2.2, we further obtain the following result:

Theorem 2.2 The part of $(\mathcal{A}, D(\mathcal{A}))$ generates a $C_{0}$-semigroup $(\mathscr{L}(X))_{t \geq 0}$ on $X_{0}$.

Since $(\mathcal{A}, D(\mathcal{A}))$ is a Hille-Yosida operator, we have the following well-posedness theorem for system (2.1).

Theorem 2.3 For any $u_{0} \in X_{0+}$, system (1.4) represented by the integral equation (2.2) has a unique continuous solution with values in $X_{0_{+}}$. Moreover, the map $\Phi:[0,+\infty) \times X_{0_{+}} \mapsto$ $X_{0+}$ defined by $\Phi\left(t, u_{0}\right)=u\left(t, u_{0}\right)$ is a continuous semiflow, that is, the map is continuous and satisfies $\Phi(0, \cdot)=I$ and $\Phi(t, \Phi(s, \cdot))=\Phi(t+s, \cdot)$.

According to the significance of epidemiology, we only consider nonnegative solutions of system (1.4). The nonnegativity and ultimate boundedness of the solution $(S(t), L(t, a), I(t))$ of system (1.4) with nonnegative initial conditions are given in the following.

Theorem 2.4 All solutions of system (1.4) with nonnegative initial conditions remain nonnegative for all $t \geq 0$ and are ultimately bounded.

Proof We first prove that $L(t, a) \geq 0$ with nonnegative initial values. Integrating the second equation in system (1.4) along the characteristic line, we obtain

$$
L(t, a)= \begin{cases}L(t-a, 0) e^{-\int_{0}^{a}(d+\alpha(\theta)) d \theta}, & a \leq t, \\ L_{0}(a-t) e^{-\int_{a-t}^{t}(d+\alpha(\theta)) d \theta}, & a>t .\end{cases}
$$

It is clear that $L(t, a)$ is nonnegative for all nonnegative initial values.

Second, we show that $I(t) \geq 0$ for $t \geq 0$. Without loss of generality, we assume there exists $t_{1}>0$ such that $I\left(t_{1}\right)=0$ and $I(t)>0$ for all $t \in\left(0, t_{1}\right)$. In fact, the third equation of system (1.4) implies that $I^{\prime}\left(t_{1}\right)=\int_{0}^{+\infty} \alpha(a) L\left(t_{1}, a\right) d a>0$. It is a contradiction, which means that the assumption is not true, that is, $I(t) \geq 0$ for all $t \geq 0$.

Third, we prove that $S(t) \geq 0$ for $t \geq 0$. In fact, if there exists $t_{2}>0$ such that $S\left(t_{2}\right)=0$ and $S(t)>0, t \in\left(0, t_{2}\right)$, then the first equation of system (1.4) implies that $S^{\prime}\left(t_{2}\right)=\Lambda+\gamma I\left(t_{2}\right)>0$, which in turn implies that $S(t) \geq 0$ for all $t \geq 0$.

Finally, we prove that the solutions of system (1.4) are ultimately bounded. Let $L(t)=$ $\int_{0}^{+\infty} L(t, a) d a$, which represents the total number of latent individuals at time $t$. It is reasonable to assume that $\lim _{a \rightarrow+\infty} L(t, a)=0$ since there exists a finite maximum age in a biological sense. Further, we have

$$
\begin{aligned}
& (S(t)+L(t)+I(t))^{\prime} \\
& \quad=\Lambda-d S(t)-\beta S(t) I(t)+\gamma I(t)-\int_{0}^{+\infty}\left(\frac{\partial L(t, a)}{\partial a}+(d+\alpha(a)) L(t, a)\right) d a
\end{aligned}
$$




$$
\begin{aligned}
& +\int_{0}^{+\infty} \alpha(a) L(t, a) d a-(d+\mu+\gamma) I(t) \\
= & \Lambda-d S(t)-\int_{0}^{+\infty}(d+\alpha(a)) L(t, a) d a+\int_{0}^{+\infty} \alpha(a) L(t, a) d a-(d+\mu) I(t) \\
= & \Lambda-d S(t)-d L(t)-(d+\mu) I(t) \\
\leq & \Lambda-d(S(t)+L(t)+I(t)) .
\end{aligned}
$$

Therefore

$$
\limsup _{t \rightarrow+\infty}(S(t)+L(t)+I(t)) \leq \frac{\Lambda}{d}
$$

Consequently, $S(t), L(t, a)$, and $I(t)$ are ultimately bounded. It follows that the omega limit set of system (1.4) is contained in the following bounded feasible region:

$$
\Gamma=\left\{(S, L(\cdot), I): S, I, L(\cdot) \geq 0, S+\int_{0}^{+\infty} L(t, a) d a+I \leq \frac{\Lambda}{d}\right\} .
$$

It is obvious that the region $\Gamma$ is positively invariant with respect to system (1.4).

\section{Equilibria and properties of linearization at equilibria}

In this part, we consider the existence of equilibria and linearization of the nonlinear system (1.4) around the equilibrium solutions.

It is clear that system (1.4) always has the disease free equilibrium $E_{0}=\left(S^{0}, 0,0\right)$, where $S^{0}=\frac{\Lambda}{d}$. In order to find the positive equilibrium $E_{*}=\left(S_{*}, L_{*}(a), I_{*}\right)$ of (1.4), we solve the following equations:

$$
\left\{\begin{array}{l}
\Lambda-d S_{*}-\beta S_{*} I_{*}+\gamma I_{*}=0, \\
\frac{d L_{*}(a)}{d a}=-(d+\alpha(a)) L_{*}(a), \\
\int_{0}^{+\infty} \alpha(a) L_{*}(a) d a-(d+\mu+\gamma) I_{*}=0, \\
L_{*}(0)=\beta S_{*} I_{*} .
\end{array}\right.
$$

Solving the second equation of (3.1), we get

$$
L_{*}(a)=L_{*}(0) e^{-\int_{0}^{a}(d+\alpha(\theta)) d \theta} .
$$

Then from the third equation of (3.1) and (3.2), we have

$$
I_{*}=\frac{L_{*}(0)}{d+\mu+\gamma} \int_{0}^{+\infty} \alpha(a) e^{-\int_{0}^{a}(d+\alpha(\theta)) d \theta} d a=\frac{\beta S_{*} I_{*}}{d+\mu+\gamma} \int_{0}^{+\infty} \alpha(a) e^{-\int_{0}^{a}(d+\alpha(\theta)) d \theta} d a .
$$

Hence

$$
S_{*}=\frac{d+\mu+\gamma}{\beta \int_{0}^{+\infty} \alpha(a) e^{-\int_{0}^{a}(d+\alpha(\theta)) d \theta} d a} .
$$


Because of $\beta S_{*}-\gamma=\frac{d+\mu+\gamma\left(1-\int_{0}^{+\infty} \alpha(a) e^{-\int_{0}^{a}(d+\alpha(\theta)) d \theta} d a\right)}{\int_{0}^{+\infty} \alpha(a) e^{-\int_{0}^{a}(d+\alpha(\theta)) d \theta} d a}>0$, the first equation of (3.1) implies that

$$
I_{*}=\frac{\Lambda}{\beta S_{*}-\gamma}\left(1-\frac{S_{*}}{S^{0}}\right) .
$$

Now we denote the basic reproduction number as

$$
R_{0}=\frac{S^{0}}{S_{*}}=\frac{\beta S^{0}}{d+\mu+\gamma} \int_{0}^{+\infty} \alpha(a) e^{-\int_{0}^{a}(d+\alpha(\theta)) d \theta} d a .
$$

Therefore, we have $I_{*}=\frac{\Lambda}{\beta S_{*}-\gamma}\left(1-\frac{1}{R_{0}}\right)>0$ when $R_{0}>1$. That is, if $R_{0}>1$, then system (1.4) has a unique positive equilibrium. Summarizing the analysis, we have the following result.

Theorem 3.1 System (1.4) always has the disease free equilibrium $E_{0}=\left(S^{0}, 0,0\right)=\left(\frac{\Lambda}{d}, 0,0\right)$. In addition, if $R_{0}>1$, then system (1.4) also has a unique endemic equilibrium $E_{*}=$ $\left(S_{*}, L_{*}(a), I_{*}\right)$, where

$$
\begin{aligned}
& S_{*}=\frac{d+\mu+\gamma}{\beta \int_{0}^{+\infty} \alpha(a) e^{-\int_{0}^{a}(d+\alpha(\theta)) d \theta} d a}, \quad I_{*}=\frac{\Lambda}{\beta S_{*}-\gamma}\left(1-\frac{1}{R_{0}}\right), \\
& L_{*}(a)=\beta S_{*} I_{*} e^{-\int_{0}^{a}(d+\alpha(\theta)) d \theta} .
\end{aligned}
$$

Here, each term in $R_{0}$ has a clear epidemiological meanings. $e^{-\int_{0}^{a}(d+\alpha(\theta)) d \theta}$ is the probability that the latent individual survives to age $a, \int_{0}^{+\infty} \alpha(a) e^{-\int_{0}^{a}(d+\alpha(\theta)) d \theta} d a$ is the total number of infectious individuals produced over the life span of the latent individual, $\frac{1}{d+\mu+\gamma}$ is the average infection period, $\beta$ is the infection rate of the infectious individual, and $\frac{\Lambda}{d}$ represents the total number of susceptible individuals. Therefore $R_{0}$ represents the total number of newly infectious individuals.

In the following, we linearize system (1.4) around the steady state. Let

$$
S(t)=x(t)+\bar{S}, \quad L(t, a)=y(t, a)+\bar{L}(a), \quad I(t)=z(t)+\bar{I},
$$

where $\bar{E}=(\bar{S}, \bar{L}(a), \bar{I})$ is any steady state of system $(1.4), \tilde{u}(t)=(x(t), y(t, a), z(t), 0)$, and $\bar{u}=$ $(\bar{S}, \bar{L}(a), \bar{I}, 0)$. Then system $(2.1)$ is equivalent to the following Cauchy problem:

$$
\left\{\begin{array}{l}
\frac{\mathrm{d}}{\mathrm{d} t} \tilde{u}(t)=\mathcal{A} \tilde{u}(t)+\mathcal{F}(\tilde{u}(t)+\bar{u})-\mathcal{F}(\bar{u}(t)), \quad t \geq 0, \\
\tilde{u}(0)=u(0)-\bar{u} .
\end{array}\right.
$$

By direct computations we can obtain readily the linearized system of (2.1) around $\bar{u}$ of the following form:

$$
\left\{\begin{array}{l}
\frac{\mathrm{d}}{\mathrm{d} t} \tilde{u}(t)=\mathcal{A} \tilde{u}(t)+D \mathcal{F}(\bar{u})(\tilde{u}(t)), \quad t \geq 0, \\
\tilde{u}(0)=u(0)-\bar{u}
\end{array}\right.
$$


where

$$
D \mathcal{F}(\bar{u})\left(\begin{array}{c}
x(t) \\
y(t, a) \\
z(t) \\
0
\end{array}\right)=\left(\begin{array}{c}
-\beta(\bar{I} x(t)+\bar{S} z(t))+\gamma z(t) \\
0 \\
\int_{0}^{+\infty} \alpha(a) y(t, a) d a \\
\beta(\bar{I} x(t)+\bar{S} z(t))
\end{array}\right) .
$$

Obviously, $D \mathcal{F}(\bar{u})$ is a compact bounded linear operator on $X$.

Combining Lemma 2.1 and Theorem 2.1, the following result can be immediately obtained:

Theorem 3.2 The operator $\mathcal{A}+D \mathcal{F}(\bar{u})$ is a Hille-Yosida operator.

Therefore, by Lemma 2.2, we can get the following result:

Theorem 3.3 The part of $(\mathcal{A}+D \mathcal{F}(\bar{u}), D(\mathcal{A}+D \mathcal{F}(\bar{u})))$ generates a $C_{0}$-semigroup $(\mathscr{T}(t))_{t \geq 0}$ on the space $X_{0}$.

By the Hille-Yosida estimate in the proof of Theorem 2.1, we have $\| \mathscr{S}(t)) \| \leq e^{-\xi t}$. Furthermore, $D \mathcal{F}(\bar{u}) \mathscr{S}(t): X_{0} \rightarrow X$ is compact for any $t>0$. Because of

$$
\mathscr{T}(t)=e^{D \mathcal{F}(\bar{u}) t} \mathscr{S}(t)=\mathscr{S}(t)+\sum_{k=1}^{+\infty} \frac{(D \mathcal{F}(\bar{u}) t)^{k}}{k !} \mathscr{S}(t),
$$

we know $(\mathscr{T}(t))_{t \geq 0}$ is quasi-compact. Theorem B.1 in [14] implies that, for some $\eta>0$, $e^{\eta t}\|\mathscr{T}(t)\| \rightarrow 0$ as $t \rightarrow+\infty$ whenever all the eigenvalues of $(\mathcal{A}+D \mathcal{F}(\bar{u}))$ have negative real parts. The previous analysis implies the following conclusion holds.

Theorem 3.4 The solution semiflow $\Phi\left(t, u_{0}\right)$ of system (1.4) defined as in Theorem 2.3 satisfies the following properties:

(i) If all the eigenvalues of $(\mathcal{A}+D \mathcal{F}(\bar{u}))$ have strictly negative real parts, then the steady state $\bar{u}$ is locally asymptotically stable.

(ii) If at least one eigenvalue of $(\mathcal{A}+D \mathcal{F}(\bar{u}))$ has a strictly positive part, then the steady state $\bar{u}$ is unstable.

\section{Stability of equilibria}

In this section, based on the preceding discussion, we will focus on discussing the stability of the disease free equilibrium $E_{0}=\left(S^{0}, 0,0\right)$ and the endemic equilibrium $E_{*}=$ $\left(S_{*}, L_{*}(a), I_{*}\right)$.

Theorem 4.1 If $R_{0}<1$, then the disease free equilibrium $E_{0}$ of system (1.4) is globally asymptotically stable.

Proof We construct the Lyapunov function to prove that $E_{0}$ is globally asymptotically stable when $R_{0}<1$. Define the Lyapunov function

$$
V(t)=S(t)-S^{0}-S^{0} \ln \frac{S(t)}{S^{0}}+m_{1} I(t)+m_{2} \int_{0}^{+\infty} \delta(a) L(t, a) d a
$$


with $m_{1}>0, m_{2}>0$, and $\delta(a)=\int_{a}^{+\infty} \alpha(\xi) e^{-\int_{a}^{\xi}(d+\alpha(\eta)) d \eta} d \xi$, where $\delta(a)$ is a differentiable function on $[0, \infty)$. Then the derivative of $V(t)$ along the solution of system (1.4) is given by

$$
\begin{aligned}
\frac{d V(t)}{d t}= & \left(1-\frac{S^{0}}{S(t)}\right) \frac{d S}{d t}+m_{1} \frac{d I}{d t}+m_{2} \int_{0}^{+\infty} \delta(a) \frac{\partial}{\partial t} L(t, a) d a \\
= & \left(1-\frac{S^{0}}{S(t)}\right)\left[d S^{0}-\beta S(t) I(t)-d S(t)+\gamma I(t)\right]+m_{1} \int_{0}^{+\infty} \alpha(a) L(t, a) d a \\
& -m_{1}(d+\mu+\gamma) I(t)-m_{2} \int_{0}^{+\infty} \delta(a)\left[\frac{\partial}{\partial t} L(t, a)+(d+\alpha(a)) L(t, a)\right] d a \\
= & \frac{-d\left(S(t)-S^{0}\right)^{2}}{S(t)}-\beta S(t) I(t)+\beta S^{0} I(t)+\gamma \frac{S(t)-S^{0}}{S(t)} I(t) \\
& +m_{1} \int_{0}^{+\infty} \alpha(a) L(t, a) d a \\
& -m_{1}(d+\mu+\gamma) I(t) n \\
& -m_{2} \int_{0}^{+\infty} \delta(a) \frac{\partial}{\partial t} L(t, a) d a-m_{2} \int_{0}^{+\infty} \delta(a)(d+\alpha(a)) L(t, a) d a .
\end{aligned}
$$

Because of

$$
\begin{aligned}
\int_{0}^{+\infty} \delta(a) \frac{\partial}{\partial t} L(t, a) d a= & \left.\delta(a) L(t, a)\right|_{a=+\infty}-\int_{0}^{+\infty} \delta^{\prime}(a) L(t, a) d a \\
= & \left.\delta(a) L(t, a)\right|_{a=+\infty}-\delta(0) L(t, 0) \\
& -\int_{0}^{+\infty}[(d+\alpha(a)) \delta(a)-\alpha(a)] L(t, a) d a
\end{aligned}
$$

(4.1) can be rewritten as

$$
\begin{aligned}
\frac{d V(t)}{d t}= & \frac{-d\left(S(t)-S^{0}\right)^{2}}{S(t)}-\beta S(t) I(t)+\beta S^{0} I(t)+\gamma \frac{S(t)-S^{0}}{S(t)} I(t) \\
& +m_{1} \int_{0}^{+\infty} \alpha(a) L(t, a) d a-m_{1}(d+\mu+\gamma) I(t)-\left.m_{2} \delta(a) L(t, a)\right|_{a=+\infty} \\
& +m_{2} \delta(0) \beta S(t) I(t)-m_{2} \int_{0}^{+\infty} \alpha(a) L(t, a) d a .
\end{aligned}
$$

It is clear that $\delta(0)=\int_{0}^{+\infty} \alpha(\xi) e^{-\int_{0}^{\xi}(d+\alpha(\eta)) d \eta} d \xi>0$. Let $m_{1}=m_{2}=\frac{1}{\delta(0)}$. Then $m_{1}=m_{2}>0$, and (4.2) can be rewritten as

$$
\begin{aligned}
\frac{d V(t)}{d t}= & \frac{-d\left(S(t)-S^{0}\right)^{2}}{S(t)}+\gamma \frac{S(t)-S^{0}}{S(t)} I(t)+I(t)\left[\beta S^{0}-m_{1}(d+\mu+\gamma)\right] \\
& -\left.m_{2} \delta(a) L(t, a)\right|_{a=+\infty} \\
= & \frac{-d\left(S(t)-S^{0}\right)^{2}}{S(t)}+\gamma \frac{S(t)-S^{0}}{S(t)} I(t)+I(t) m_{1}(d+\mu+\gamma)\left(R_{0}-1\right) \\
& -\left.m_{2} \delta(a) L(t, a)\right|_{a=+\infty} .
\end{aligned}
$$


Since $0<S(t) \leq \frac{\Lambda}{d}=S^{0}$ for $(S, L(\cdot), I) \in \Gamma$, when $R_{0}<1$ and $m_{1}=\frac{1}{\delta(0)}$, the function $V$ and its derivative along solutions of model (1.4) are positive and negative on the set $\Gamma$, respectively. Therefore the Lyapunov stability theorem implies that the disease-free equilibrium $E_{0}$ of system (1.4) is globally asymptotically stable on the set $\Gamma$ when $R_{0}<1$ [15].

Theorem 4.2 If $R_{0}>1$, then the endemic equilibrium $E_{*}$ of system (1.4) is locally asymptotically stable.

Proof To explore the local stability of $E_{*}$, we linearize system (1.4) at endemic equilibrium $E_{*}$. Namely, we introduce the perturbation variables $x(t)=S(t)-S_{*}, y(t, a)=L(t, a)-L_{*}(a)$, and $z(t)=I(t)-I_{*}$, which leads to

$$
\left\{\begin{array}{l}
x^{\prime}(t)=-d x(t)-\beta\left(I_{*} x(t)+S_{*} z(t)\right)+\gamma z(t), \\
\frac{\partial y(t, a)}{\partial t}+\frac{\partial y(t, a)}{\partial a}=-(d+\alpha(a)) y(t, a), \\
z^{\prime}(t)=\int_{0}^{+\infty} \alpha(a) y(t, a) d a-(d+\mu+\gamma) z(t), \\
y(t, 0)=\beta\left(I_{*} x(t)+S_{*} z(t)\right) .
\end{array}\right.
$$

We substitute $x(t)=x_{0} e^{\lambda t}, y(t, a)=y_{0}(a) e^{\lambda t}$, and $z(t)=z_{0} e^{\lambda t}$ into equation (4.4) and get the following equations:

$$
\left\{\begin{array}{l}
(\lambda+d) x_{0}=-\beta\left(I_{*} x_{0}+S_{*} z_{0}\right)+\gamma z_{0}, \\
\frac{d y_{0}(a)}{d a}=-(\lambda+d+\alpha(a)) y_{0}(a), \\
(\lambda+d+\mu+\gamma) z_{0}=\int_{0}^{+\infty} \alpha(a) y_{0}(a) d a, \\
y_{0}(0)=\beta\left(I_{*} x_{0}+S_{*} z_{0}\right) .
\end{array}\right.
$$

Solving the second and the third equations in (4.5), we get

$$
\begin{aligned}
& y_{0}(a)=y_{0}(0) e^{-\int_{0}^{a}(\lambda+d+\alpha(\theta)) d \theta} \text { and } \\
& z_{0}=\frac{y_{0}(0)}{\lambda+d+\mu+\gamma} \int_{0}^{+\infty} \alpha(a) e^{-\int_{0}^{a}(\lambda+d+\alpha(\theta)) d \theta} d a .
\end{aligned}
$$

By the first equation in (4.5), combining the last equation in (4.5), we obtain

$$
(\lambda+d) x_{0}=-y_{0}(0)+\gamma z_{0}, \quad\left(\lambda+d+\beta I_{*}\right) x_{0}=\left(r-\beta S_{*}\right) z_{0} .
$$

Then we derive the characteristic equation of (4.5) as follows:

$$
\frac{\lambda+d+\beta I_{*}}{\lambda+d}=\frac{\left(\gamma-\beta S_{*}\right) z_{0}}{\gamma z_{0}-y_{0}(0)}=\frac{\left(\gamma-\beta S_{*}\right)}{\gamma-\frac{y_{0}(0)}{z_{0}}} .
$$

It is not hard to see that for $\lambda$ with $\operatorname{Re} \lambda \geq 0$, the left side of the characteristic equation satisfies

$$
\left|\frac{\lambda+d+\beta I_{*}}{\lambda+d}\right|>1
$$




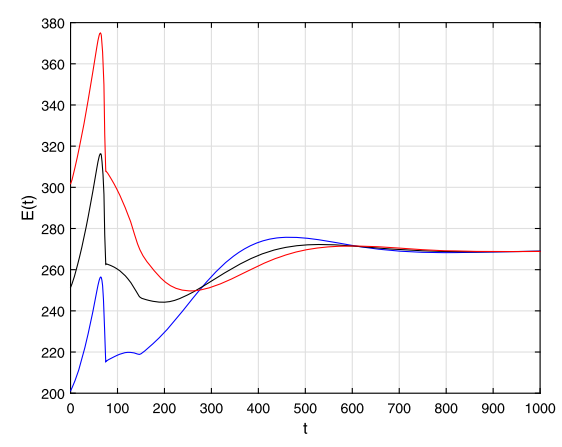

(a) The number of latent individuals

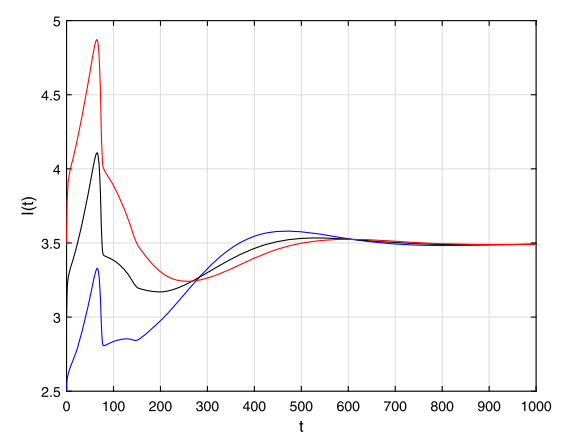

(b) The number of infectious individuals

Figure 1 The global asymptotical stability of the endemic equilibrium $E_{*}$ when $R_{0}>1$, where $E(t)$ represents the latent individuals, and $/(t)$ represents the infectious individuals

On the other hand, for the right side of (4.8), we have for $\lambda$ with $\operatorname{Re} \lambda \geq 0$ :

$$
\frac{y_{0}(0)}{z_{0}}=\frac{\lambda+d+\mu+\gamma}{\int_{0}^{+\infty} \alpha(a) e^{-\int_{0}^{a}(\lambda+d+\alpha(\theta)) d \theta} d a}>\frac{d+\mu+\gamma}{\int_{0}^{+\infty} \alpha(a) e^{-\int_{0}^{a}(d+\alpha(\theta)) d \theta} d a}=\beta S_{*}>\gamma,
$$

which implies that $\left|\frac{\left(\gamma-\beta S_{*}\right)}{\gamma-\frac{y_{0}(0)}{z_{0}}}\right|=\left|\frac{\left(\beta S_{*}-\gamma\right)}{\frac{y_{0}(0)}{z_{0}}-\gamma}\right| \leq 1$. It is a contradiction since the roots of the characteristic equation (4.8) must have negative real parts. Therefore the endemic equilibrium $E_{*}$ is locally asymptotically stable when $R_{0}>1$.

It is so difficult to prove the global stability of $E_{*}$ by the rigorous mathematical theory. Therefore, we will use the numeric simulations to disply the endemic equilibrium $E_{*}$ may be globally asymptotically stable when $R_{0}>1$. In order to achieve this goal, we take the maximum infection age $75, \Lambda=10, d=0.007, \mu=0.0025, \gamma=0.75, \tau=0.5$, and $\alpha_{*}=0.01$. With these parameter values, by straightforward calculations, we have $R_{0}=2.2051>1$ when $\beta=0.002$. Figure 1 displays that the solutions of system (1.4) with three different initial values tend to the endemic equilibrium $E_{*}$ as $t$ tends to infinity. It implies that $E_{*}$ may be globally stable when $R_{0}>1$.

\section{Uniform persistence of infection}

Although we are not able to prove that $E_{*}$ is globally asymptotically stable when $R_{0}>1$ by using the strict mathematical derivation, we can prove that the persistence of system (1.4) when $R_{0}>1$. In this section, we will investigate the persistence of system (1.4) by using the method in [16]. We give the following notations:

$$
\begin{aligned}
& \mathcal{M}=\left\{\varphi(a) \in L_{+}^{1}(0,+\infty) \mid \exists t \geq 0 \text { such that } \int_{0}^{+\infty} \alpha(a+t) \varphi(a) d a>0\right\} \\
& \Gamma_{0}=\mathbb{R}_{+} \times \mathcal{M} \times \mathbb{R}_{+}, \Upsilon=\Gamma \cap \Gamma_{0} .
\end{aligned}
$$

We first prove that system (1.4) is uniformly weakly persistent. To this end, we give the following result. 
Lemma 5.1 If $R_{0}>1$, then there exists a positive constant $\varepsilon_{0}>0$ such that any solution $(S(t), L(t, a), I(t))$ of system (1.4) with initial values $\left(S_{0}, L_{0}(a), I_{0}\right) \in \Upsilon$ satisfies

$$
\limsup _{t \rightarrow+\infty} I(t)>\varepsilon_{0}
$$

Proof For any solution $(S(t), L(t, a), I(t))$ of system (1.4) with initial value $\left(S_{0}, L_{0}(a), I_{0}\right) \in \Upsilon$, we claim that (5.1) holds. Otherwise, for any $\varepsilon>0$, there exists a sufficiently large constant $t_{1}>0$ such that $I(t) \leq \varepsilon$ for all $t \geq t_{1}$.

The first equation of system (1.4) implies that

$$
\frac{d S(t)}{d t} \geq \Lambda-d S(t)-\beta S(t) I(t) \geq \Lambda-d S(t)-\beta S(t) \varepsilon=\Lambda-(d+\beta \varepsilon) S(t) .
$$

That is, $\limsup _{t \rightarrow+\infty} S(t) \geq \liminf _{t \rightarrow+\infty} S(t) \geq \frac{\Lambda}{d+\beta \varepsilon}$.

Let $\phi(t)=L(t, 0)$, which satisfies

$$
\phi(t) \geq \frac{\beta \Lambda}{d+\beta \varepsilon} I(t) \quad \text { for } t \geq t_{1} .
$$

Combining (2.4) and the third equation of (1.4), we have

$$
I^{\prime}(t) \geq \int_{0}^{t} \alpha(a) \phi(t-a) e^{-\int_{0}^{a}(d+\alpha(\theta)) d \theta} d a-(d+\mu+\gamma) I(t) .
$$

Taking the Laplace transforms of both sides of (5.2) and (5.3), for $s \in \mathbb{R}$, we have,

$$
\begin{aligned}
& \hat{\phi}(s) \geq \frac{\beta \Lambda}{d+\beta \varepsilon} \hat{I}(s), \\
& s \hat{I}(s)-I(0) \geq \hat{K}(s) \hat{\phi}(s)-(d+\mu+\gamma) \hat{I}(s),
\end{aligned}
$$

where $\hat{\phi}(s)$ and $\hat{I}(s)$ represent the Laplace transforms of $\phi$ and $I$, respectively, and

$$
\hat{K}(s)=\int_{0}^{+\infty} \alpha(a) e^{-\int_{0}^{a}(d+\alpha(\theta)) d \theta} e^{-s a} d a .
$$

Combining (5.4) and (5.5), we get

$$
\hat{\phi}(s) \geq \frac{\beta \Lambda \hat{K}(s)}{(d+\beta \varepsilon)(s+d+\mu+\gamma)} \hat{\phi}(s)+\frac{\beta \Lambda}{(d+\beta \varepsilon)(s+d+\mu+\gamma)} I(0) .
$$

Denote $H(\varepsilon, s)=\frac{\beta \Lambda \hat{K}(s)}{(d+\beta \varepsilon)(s+d+\mu+\gamma)}$, we have

$$
\lim _{\varepsilon, s \rightarrow 0} H(\varepsilon, s)=\frac{\beta \Lambda}{d(d+\mu+\gamma)} \int_{0}^{+\infty} \alpha(a) e^{-\int_{0}^{a}(d+\alpha(\theta)) d \theta} d a=R_{0}>1,
$$

which is a contradiction since the coefficient of $\hat{\phi}(s)$ on the right-hand side of (5.6) should be less than 1 . Therefore, the assumption is not true. That is, there exists a constant $\varepsilon_{0}>0$, 
such that any solution $(S(t), L(t, a), I(t))$ of $(1.4)$ with $\left(S_{0}, L_{0}(a), I_{0}\right) \in \Upsilon$ satisfies

$$
\limsup _{t \rightarrow+\infty} I(t)>\varepsilon_{0}
$$

Further, by Lemma 5.1, we know any solution $(S(t), L(t, a), I(t))$ of system (1.4) is bounded below. In fact, the definition of $\Upsilon$ implies that $I(t)$ is bounded above. Let $I(t) \leq M$. Then

$$
\frac{d S(t)}{d t} \geq \Lambda-d S(t)-\beta S(t) I(t) \geq \Lambda-d S(t)-\beta M S(t)
$$

which implies that

$$
\limsup _{t \rightarrow+\infty} S(t) \geq \liminf _{t \rightarrow+\infty} S(t) \geq \frac{\Lambda}{d+\beta M}
$$

Then, by using (5.7) and (5.8), we have

$$
\limsup _{t \rightarrow+\infty} \phi(t) \geq \frac{\beta \Lambda \varepsilon_{0}}{d+\beta M}
$$

from which we infer that

$$
\limsup _{t \rightarrow+\infty} \int_{0}^{+\infty} L(t, a) d a \geq \frac{\beta \Lambda \varepsilon_{0}}{d+\beta M} \int_{0}^{+\infty} e^{-\int_{0}^{a}(d+\alpha(\theta)) d \theta} d a>0 .
$$

Combining Lemma 5.1, (5.8), and (5.9) yields immediately the weak persistence of the solutions to system (1.4). Namely, we have the following result:

Theorem 5.1 If $R_{0}>1$, then the semiflow $\{\Phi(t)\}_{t \geq 0}$ generated by system (1.4) is weakly persistent in $\Upsilon$ in the sense that there exists a constant $\zeta>0$, such that

$$
\limsup _{t \rightarrow+\infty} S(t) \geq \zeta, \quad \limsup _{t \rightarrow+\infty}\|L(t, a)\|_{L_{1}} \geq \zeta, \quad \limsup _{t \rightarrow+\infty} I(t) \geq \zeta
$$

Next, we will use the methods and techniques recently employed in $[14,17,18]$ to show that system (1.4) has a global attractor $\mathscr{A}_{0}$. Namely, the following result holds.

Lemma 5.2 If $R_{0}>1$, then there exists a global attractor $\mathscr{A}_{0} \subseteq \Upsilon$ for the solution semiflow $(\Phi(t))_{t \geq 0}$ of system (1.4).

Proof It is clear that the semiflow $(\Phi(t))_{t \geq 0}$ defined in Theorem 2.3 maps $\mathbb{R}_{+} \times \Upsilon$ into $\Upsilon$, and $(\Phi(t))_{t \geq 0}$ is point dissipative and maps bounded sets into bounded sets.

We decompose the solution semiflow $\Phi$ as $\Phi=\Psi\left(t, u_{0}\right)+\Theta\left(t, u_{0}\right)$ with

$$
\Psi\left(t, u_{0}\right)=\left(0, L_{1}(t, \cdot), 0\right) \quad \text { and } \quad \Theta\left(t, u_{0}\right)=\left(S(t), L_{2}(t, \cdot), I(t)\right)
$$


where $S(t)$ and $I(t)$ satisfy system (1.4), and $L_{1}(t, a)$ and $L_{2}(t, a)$ are respectively the solutions of the systems

$$
\left\{\begin{array}{l}
\frac{\partial L_{1}(t, a)}{\partial t}+\frac{\partial L_{1}(t, a)}{\partial a}=-(d+\alpha(a)) L_{1}(t, a) \\
L_{1}(t, 0)=0 \\
L_{1}(0, a)=L_{0}(a)
\end{array}\right.
$$

and

$$
\left\{\begin{array}{l}
\frac{\partial L_{2}(t, a)}{\partial t}+\frac{\partial L_{2}(t, a)}{\partial a}=-(d+\alpha(a)) L_{2}(t, a) \\
L_{2}(t, 0)=\beta S(t) I(t) \\
L_{2}(0, a)=0
\end{array}\right.
$$

It is easy to check that $L_{1}(t, a)$ and $L_{2}(t, a)$ are both nonnegative. Let $\bar{w}(t)=\int_{0}^{+\infty} L_{1}(t, a) d a$. Then (5.10) implies that $\bar{w}(t) \leq\left\|L_{0}(a)\right\|_{L^{1}} e^{-d t}$, and hence $\lim _{t \rightarrow+\infty} \Psi\left(t, u_{0}\right)=0$ for every $u_{0} \in \Upsilon$.

To prove that $\Theta\left(t, u_{0}\right)$ is completely continuous, we show that the set $\left\{\Theta\left(t, u_{0}\right): u_{0} \in\right.$ $\Xi$ \} is precompact for fixed $t$ and any bounded set $\Xi$ in $\Upsilon$. To this end, we only need to verify that the set $\left\{\Theta\left(t, u_{0}\right): u_{0} \in \Upsilon\right\}$ is precompact by utilizing the Fréchet-Kolmogorov theorem. Obviously, because of $\left\{\Theta\left(t, u_{0}\right): u_{0} \in \Upsilon\right\} \subseteq \Upsilon$, we obtain that $\left\{\Theta\left(t, u_{0}\right): u_{0} \in \Upsilon\right\}$ is bounded. In addition, by (5.11) we have

$$
L_{2}(t, a)= \begin{cases}\check{\phi}(t-a) e^{-\int_{0}^{a}(d+\alpha(\theta)) d \theta}, & a \leq t, \\ 0, & a>t,\end{cases}
$$

where $\check{\phi}(t)=L_{2}(t, 0)=\beta S(t) I(t)$. Then we have $L_{2}(t, a)=0$ for $a>t$.

Next, we show that the $L^{1}$-norm of $\frac{\partial L_{2}(t, a)}{\partial a}$ is bounded. Indeed, since, for $u_{0} \in \Upsilon, \check{\phi}(t), S(t)$ and $I(t)$ are all bounded, from the first and third equations of system (1.4) we deduce that $\left|\check{\phi}^{\prime}(t)\right|=\beta\left|S^{\prime}(t) I(t)+S(t) I^{\prime}(t)\right|$ is bounded as well. Then we have $\check{\phi}(t) \leq \delta_{1}$ and $\left|\check{\phi}^{\prime}(t)\right| \leq \delta_{2}$, where $\delta_{1}$ and $\delta_{2}$ are two constants depending on the parameters and the bounds of the solutions. From (5.12) we derive that

$$
\frac{\partial L_{2}(t, a)}{\partial a}= \begin{cases}-\check{\phi}^{\prime}(t-a) e^{-\int_{0}^{a}(d+\alpha(\theta)) d \theta}-\check{\phi}(t-a) \alpha(a) e^{-\int_{0}^{a}(d+\alpha(\theta)) d \theta}, & a \leq t, \\ 0, & a>t\end{cases}
$$

and then we obtain that

$$
\begin{aligned}
\left\|\frac{\partial L_{2}(t, a)}{\partial a}\right\|_{L^{1}} \leq & \int_{0}^{+\infty}\left|\check{\phi}^{\prime}(t-a)\right| e^{-\int_{0}^{a}(d+\alpha(\theta)) d \theta} d a \\
& +\int_{0}^{+\infty} \check{\phi}(t-a) \alpha(a) e^{-\int_{0}^{a}(d+\alpha(\theta)) d \theta} d a \leq \epsilon,
\end{aligned}
$$

that is, $\frac{\partial L_{2}(t, a)}{\partial a}$ is bounded in $L^{1}$. Thus, observing that

$$
\int_{0}^{+\infty}\left|L_{2}(t, a+\theta)-L_{2}(t, a)\right| d a \leq\left\|\frac{\partial L_{2}(t, a)}{\partial a}\right\|_{L^{1}} \cdot|\theta| \leq \epsilon|\theta| \rightarrow 0 \quad \text { as } \theta \rightarrow 0,
$$


we infer that, for a fixed $t,\left\{\Theta\left(t, u_{0}\right): u_{0} \in \Upsilon\right\}$ is precompact. So, by the FréchetKolmogorov theorem $\Theta\left(t, u_{0}\right)$ is completely continuous. Thus all the conditions of Theorem 3.4.6 in [17] are satisfied, and the result follows. The proof is completed.

Based on the pevious analysis, we get the following result.

Lemma 5.3 If $R_{0}>1$, then there exists a positive constant $\eta>0$ such that any solution $(S(t), L(t, a), I(t))$ of (1.4) with initial value $\left(S_{0}, L_{0}(a), I_{0}\right) \in \Upsilon$ satisfies

$$
\liminf _{t \rightarrow+\infty} I(t)>\eta
$$

Proof We consider the solution semiflow $\Phi$ on $\Upsilon$. Define the function $\rho: \Upsilon \mapsto \mathbb{R}_{+}$by

$$
\rho\left(\Phi\left(t, u_{0}\right)\right)=I(t)
$$

Lemma 5.1 implies that the semiflow is uniformly weakly $\rho$-persistent, and Lemma 5.2 means that the solution semiflow has a global attractor $\mathscr{A}_{0}$. Since the solution semiflow is nonnegative for all $t \geq 0$, by the the third equation of system (1.4) we know that $I(t) e^{(d+\mu+\gamma) t}$ is increasing. Therefore, for any $s$ with $t>s$, we have

$$
I(t) \geq I(s) e^{-(d+\mu+\gamma)(t-s)}
$$

that is, $I(t)>0$ for all $t>s$ when $I(s)>0$. It then follows from Theorem 2.6 in [16] that the solution semiflow is uniformly strongly $\rho$-persistent, thats is, there exists a constant $\eta>0$ such that

$$
\liminf _{t \rightarrow+\infty} I(t)>\eta
$$

By (5.8) we can get that

$$
\liminf _{t \rightarrow+\infty} L(t, 0)=\liminf _{t \rightarrow+\infty} \beta S(t) I(t) \geq \frac{\beta \Lambda \eta}{d+\beta M} .
$$

Thus $E(t, 0)$ is uniformly strongly persistent.

It is easy to show that the persistence (i.e., with respect to $L(t, 0))$ implies the persistence of $L(t, \cdot)$ with respect to $\|\cdot\|_{L^{1}}$. In fact, by a variation of the Lebesgue-Fatou lemma ([19], Section B.2) we obtain

$$
\begin{aligned}
\liminf _{t \rightarrow+\infty}\|L(t, \cdot)\|_{L^{1}} & \geq \int_{0}^{+\infty} \liminf _{t \rightarrow+\infty} L(t, a) d a \\
& =\int_{0}^{+\infty} \liminf _{t \rightarrow+\infty} L(t-a, 0) e^{-\int_{0}^{a}(d+\alpha(\theta)) d \theta} d a \\
& \geq \frac{\beta \Lambda \eta}{d+\beta M} \int_{0}^{+\infty} e^{-\int_{0}^{a}(d+\alpha(\theta)) d \theta} d a .
\end{aligned}
$$

Hence the persistence of $L(t, a)$ with respect to $\|\cdot\|_{L^{1}}$ follows. Then, combining Lemmas 5.2 and 5.3, we have the following theorem. 
Theorem 5.2 If $R_{0}>1$, then the semiflow $\{\Phi(t)\}_{t \geq 0}$ generated by (1.4) is uniformly persistent in $\Upsilon$ in the sense that there exists a constant $\zeta>0$ such that

$$
\liminf _{t \rightarrow+\infty} S(t) \geq \zeta, \quad \liminf _{t \rightarrow+\infty}\|L(t, a)\|_{L_{1}} \geq \zeta, \quad \liminf _{t \rightarrow+\infty} I(t) \geq \zeta
$$

\section{Conclusion}

In this paper, we have proposed and analyzed a SLIS epidemic model with age-structure and delay. Firstly, we showed that the well-posedness of system (1.4) and solutions of the system are positive and ultimately bounded. Then we derived the basic reproduction number $R_{0}$ and proved that it is the threshold to determine extinction or survival of viruses. Precisely, a disease-free equilibrium is globally asymptotically stable if $R_{0}<1$ and unstable if $R_{0}>1$. Moreover, disease persists in the latter case in the sense that the infectious individuals survive above a certain number for any initial infection number. We further explored the local stability of endemic equilibrium by analyzing the distribution of roots to the related characteristic equation. Numerical simulations display that the endemic equilibrium may be globally stable.

The mathematical model we build in this paper can be used to describe the transmission of TB, hand-foot-mouth disease, certain types of computer viruses, and so on. If data on the transmission of a certain type of infectious disease are plentiful, then we are able to use our model to even predict the number of infected individuals with different infection ages. This information has a certain guiding significance for the public health department or information security department to formulate relevant prevention and control measures for the transmission of disease or computer viruses.

The challenge we face in the future is constructing a reasonable Lyapunov function to prove the global stability of the endemic equilibrium of epidemic model with agestructure. In addition, in this paper, we choose the progression rate $\alpha(a)$ as a general function $\alpha_{*}(a)$ when $a \geq \tau$. In fact, choosing a proper function form for $\alpha_{*}(a)$ is also a meaningful question in the future.

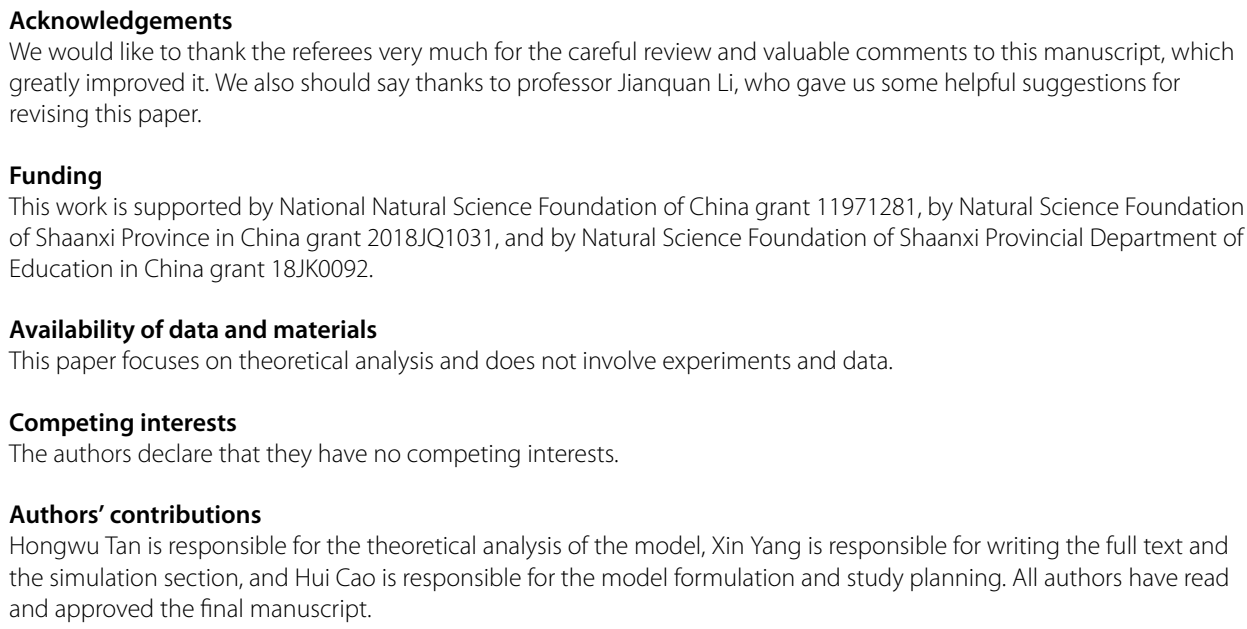

This work is supported by National Natural Science Foundation of China grant 11971281, by Natural Science Foundation of Shaanxi Province in China grant 2018JQ1031, and by Natural Science Foundation of Shaanxi Provincial Department of Education in China grant 18JK0092.

Availability of data and materials

This paper focuses on theoretical analysis and does not involve experiments and data.

Competing interests

The authors declare that they have no competing interests.

Authors' contributions

Hongwu Tan is responsible for the theoretical analysis of the model, Xin Yang is responsible for writing the full text and the simulation section, and Hui Cao is responsible for the model formulation and study planning. All authors have read and approved the final manuscript.

\section{Publisher's Note}

Springer Nature remains neutral with regard to jurisdictional claims in published maps and institutional affiliations. 


\section{References}

1. Kermack, W.O., McKendrick, A.G.: A contribution to the mathematical theory of epidemics. Proc. R. Soc. A. 115, 700-721 (1927)

2. Li, M.Y., Muldoweney, J.S.: Global stability for SEIR model in epidemiology. Math. Biosci. 125, 155-167 (1995)

3. Li, M.Y., Muldowney, J.S., Wang, L.C., Karsai, J.: Global dynamics of an SEIR epidemic model with a varying total population size. Math. Biosci. 160, 191-213 (1999)

4. Li, M.Y., Muldowney, J.S.: Global stability of a SEIR epidemic model with vertical transmission. SIAM J. Appl. Math. 62, 58-69 (2001)

5. Zhang, J., Ma, Z.: Global stability of SEIR model with saturating contact rate. Math. Biosci. 185, 15-32 (2003)

6. Xu, R., Wang, Z., Zhang, F.: Global stability and Hopf bifurcations of an SEIR epidemiological model with logistic growth and time delay. Appl. Math. Comput. 269, 332-342 (2015)

7. Britton, T., Trapman, P.: Stochastic epidemics in growing populations. Bull. Math. Biol. 76, 985-996 (2014)

8. Sharma, N., Gupta, A.K.: Impact of time delay on the dynamics of SEIR epidemic model using cellular automata. Phys. A, Stat. Mech. Appl. 471, 114-125 (2017)

9. Anderson, R.M., May, R.M.: Population biology of infectious diseases: part 1. Nature 280, 361 (1979)

10. Fan, M., Li, M.Y., Wang, K.: Global stability of an SEIS epidemic model with recruitment and a varying total population size. Math. Biosci. 170, 199-208 (2001)

11. Yan, D., Fu, X.: Analysis of an age-structured HIV infection model with logistic target-cell growth and antiretroviral therapy. IMA J. Appl. Math. 83, 1037-1065 (2018)

12. Engel, K.J., Nagel, R.: One-Parameter Semigroups for Linear Evolution Equations. Springer, New York (2000)

13. Pazy, A.: Semigroups of Linear Operators and Applications to Partial Differential Equations. Springer, New York (1983)

14. Martcheva, M., Thieme, H.R.: Progression age enhanced backward bifurcation in an epidemic model with super-infection. J. Math. Biol. 46, 385-424 (2003)

15. Hale, J.K.: Theory of Functional Differential Equations. Springer, Heidelberg (1977)

16. Thieme, H.R.: Uniform persistence for non-autonomous semiflows in population biology. Math. Biosci. 166, 173-201 (2000)

17. Hale, J.K.: Asymptotic Behavior of Dissipative Systems. Math. Surveys Monogr., vol. 25. AMS, Providence (1988)

18. Martcheva, M., Li, X.Z.: Competitive exclusion in an infection-age structured model with environmental transmission. J. Math. Anal. Appl. 408, 225-246 (2013)

19. Smith, H.L.: Mathematics in Population Biology. Princeton University Press, Princeton (2003)

\section{Submit your manuscript to a SpringerOpen ${ }^{\circ}$ journal and benefit from:}

- Convenient online submission

- Rigorous peer review

- Open access: articles freely available online

- High visibility within the field

- Retaining the copyright to your article

Submit your next manuscript at $>$ springeropen.com 